Article

\title{
Derivate Isocorydine (d-ICD) Suppresses Migration and Invasion of Hepatocellular Carcinoma Cell by Downregulating ITGA1 Expression
}

\author{
Xiaoqin Liu, Hua Tian, Hong Li, Chao Ge, Fangyu Zhao, Ming Yao and Jinjun Li * \\ State Key Laboratory of Oncogenes and Related Genes, Shanghai Cancer Institute, Renji Hospital, \\ Shanghai Jiaotong University School of Medicine, 25/Ln 2200, Xietu Road, Shanghai 200032, China; \\ liuxiaoqin106484@163.com (X.L.); htian@shsci.org (H.T.); hongli@shsci.org (H.L.); chaoge1127@163.com (C.G.); \\ fangyuzhao11@163.com (F.Z.); myao@shsci.org (M.Y.) \\ * Correspondence: jjli@shsci.org; Tel./Fax: +86-21-6443-2140
}

Academic Editor: William Chi-shing Cho

Received: 24 January 2017; Accepted: 22 February 2017; Published: 27 February 2017

\begin{abstract}
In our previous studies, we found that isocorydine (ICD) could be a potential antitumor agent in hepatocellular carcinoma (HCC). Derivate isocorydine (d-ICD), a more effective antitumor agent, has been demonstrated to inhibit proliferation and drug resistance in HCC. In order to investigate the potential role of d-ICD on HCC cell migration and its possible mechanism, wound healing assay, trans-well invasion assay, western blot analysis, and qRT-PCR were performed to study the migration and invasion ability of HCC cells as well as relevant molecular alteration following d-ICD treatment. Results indicated that the migration and invasion ability of HCC cells were suppressed when cultured with d-ICD. Meanwhile, the expression level of ITGA1 was markedly reduced. Furthermore, we found that ITGA1 promotes HCC cell migration and invasion in vitro, and that ITGA1 can partly reverse the effect of d-ICD-induced migration and invasion suppression in HCC cells. In addition, dual luciferase reporter assay and chromatin immunoprecipitation assay were used to study the expression regulation of ITGA1, and found that E2F1 directly upregulates ITGA1 expression and d-ICD inhibits E2F1 expression. Taken together, these results reveal that d-ICD inhibits HCC cell migration and invasion may partly by downregulating E2F1/ITGA1 expression.
\end{abstract}

Keywords: d-ICD; ITGA1; migration; invasion; hepatocellular carcinoma

\section{Introduction}

Liver cancer is the second leading cause of cancer death worldwide, and most (70\% to $90 \%$ ) pathological types of liver cancers are hepatocellular carcinoma (HCC) [1]. The main cause of cure difficulty in HCC patients is the lack of effective drugs for tumor recurrence and metastasis [2]. Thus, the need for developing an effective antineoplastic for metastatic HCC is extremely urgent.

Isocorydine (ICD), as an alkaloid, is purified from Papaveraceae sp. plants, such as Dactylicapnos scandens Hutchins and Dicranostigma leptopodum (Maxim.) Fedde. It is used as a clinic drug for the treatment of spasmolytic, vasodilating, antiplasmodial, and antiarrhythmic symptoms. Our previous studies demonstrated that ICD inhibited HCC growth by inducing cell cycle arrest and apoptosis of HCC cells [3], and significantly decreasing the percentage of side population (SP) cells and sensitizing cancer cells to chemical therapy in HCC cell lines [4]. However, the effective dosage of ICD for anti-HCC reached up to $200 \mu \mathrm{M}$, which has been proposed to be too high a dosage for clinical treatment. Derivate isocorydine (d-ICD), the chemical structure of ICD modified at C-8 (8-amino-isocorydine) [4,5], has proved to be more stable and effective regarding anticancer activity [5]. Our previous studies showed that d-ICD suppressed HCC cell growth both in vitro and in vivo and 
inhibited drug resistance at a lower dosage [6,7]. However, whether or not the more effective anti-HCC agent d-ICD has an effect on the migration and invasion of HCC cells is still unknown. In this study, we investigated the effects of d-ICD on HCC cell migration and invasion, as well as the underlying molecular mechanism.

Integrin-mediated adhesion of cell-to-cell and cell-to-extracellular matrix and integrin dysregulation have been proposed to partly contribute to tumor progression and metastasis [8-11]. As one of the integrin family members, integrin alpha1, which is encoded by the ITGA1 gene, was first investigated in immune disease [12]. Further studies have suggested that integrin alpha1 is closely associated with angiogenesis $[13,14]$. Recent studies have revealed that ITGA1 is upregulated in higher metastasis potential melanoma cells, gastric cancer cells, and colorectal cancer cells [15-17]. Blocking integrin $\alpha 1$ with a therapeutic monoclonal antibody or knocking out ITGA1 in mice both indicated that ITGA1 promoted tumor cell migration and invasion [18-20]. However, the specific role of ITGA1 in HCC has not been fully understood.

E2F1 is a well-known transcriptional factor that widely regulates gene expression that is involved in proliferation, autophagy, and apoptosis [21,22]. The biological function of $E 2 F 1$ has been shown to be controversial during HCC tumorigenesis $[23,24]$. In our present study, we found that $E 2 F 1$ may also upregulate ITGA1 expression in HCC.

In the present study, we found that d-ICD treatment inhibited HCC cell migration and invasion in vitro, and that inhibition was partly achieved by downregulating E2F1/ITGA1 expression. Taken together, our results further proved that d-ICD may be a more effective anti-tumor agent in HCC.

\section{Results}

\section{1. d-ICD Inhibits HCC Cell Migration and Invasion In Vitro and Downregulates ITGA1 Expression}

We performed the wound healing assay and trans-well assay with or without d-ICD treatment in HCC cells. Different types of HCC cell lines vary in sensitivity to d-ICD and the d-ICD concentration according to a previous study in our laboratory [6,7]. The results showed that d-ICD inhibited HCC cell migration and invasion (Figure 1A,B). To further elucidate the molecular mechanism involved in this phenomenon, we analyzed the differential gene expression obtained from the cDNA microarrays of HCC cell lines SMMC-7721 and Huh7 between the d-ICD treated HCC cells and control group obtained from a previously published study [7]. Combined with the fold changes and biological function of the differentially expressed genes, we finally targeted against ITGA1 and confirmed the downregulation of ITGA1 in HCC cell lines Huh7, SMMC-7721, MHCC-97L, and MHCC-LM3 via qRT-PCR and western blotting analyses (Figure 1C). Next, we analyzed the intrinsic ITGA1 expression in HCC cell lines using qRT-PCR and western blotting analyses (Figure 1D and Figure S1).

$\mathbf{A}$

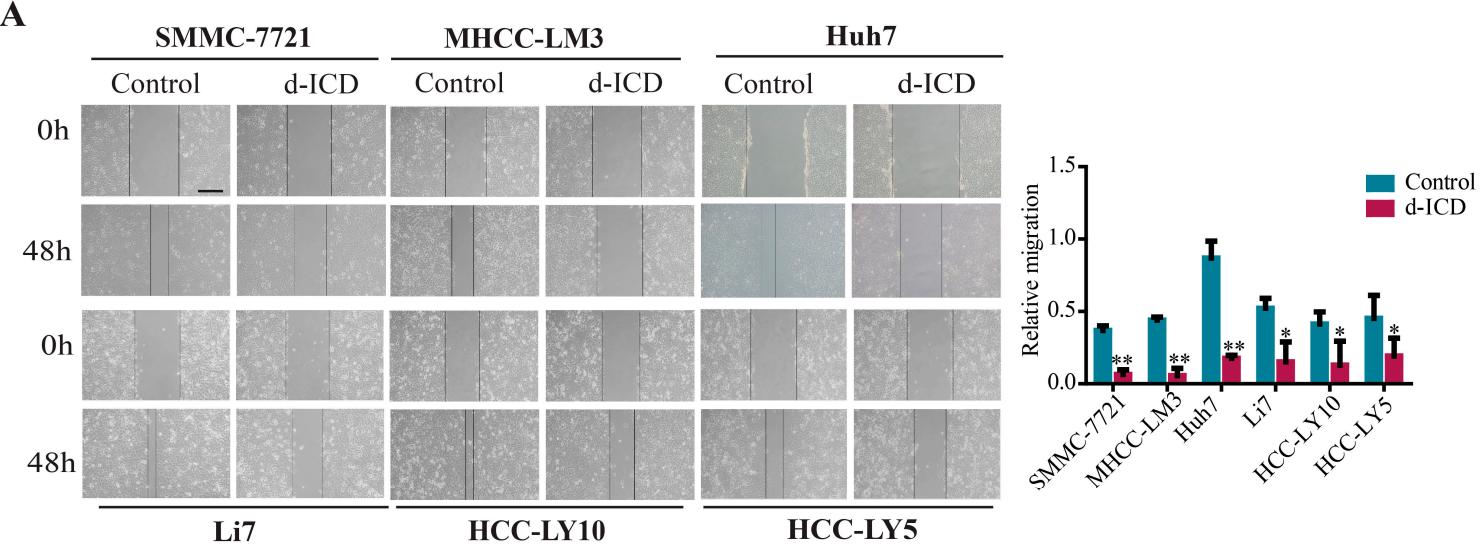

Figure 1. Cont. 
B
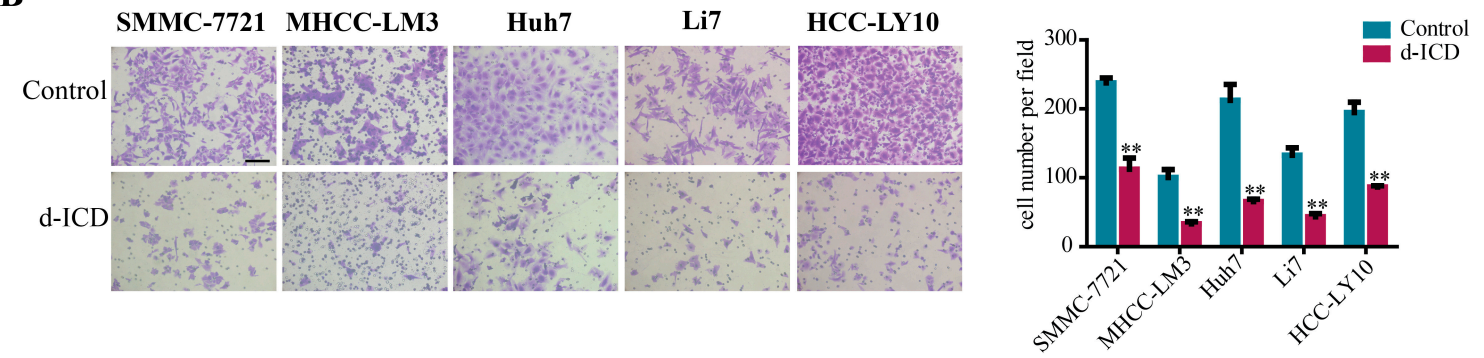

C
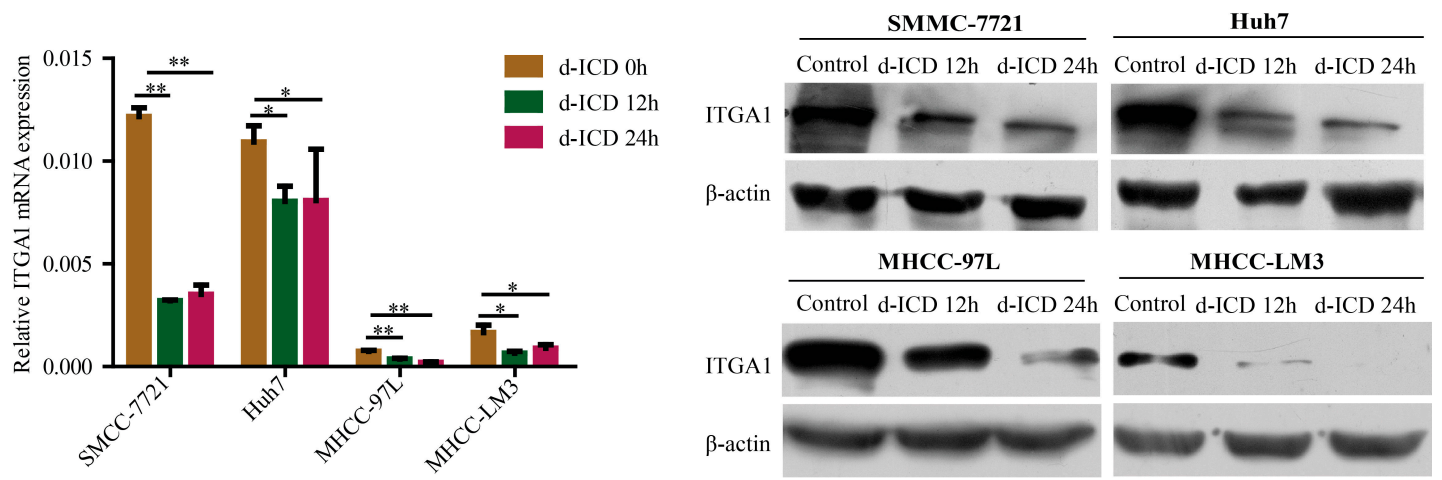

D

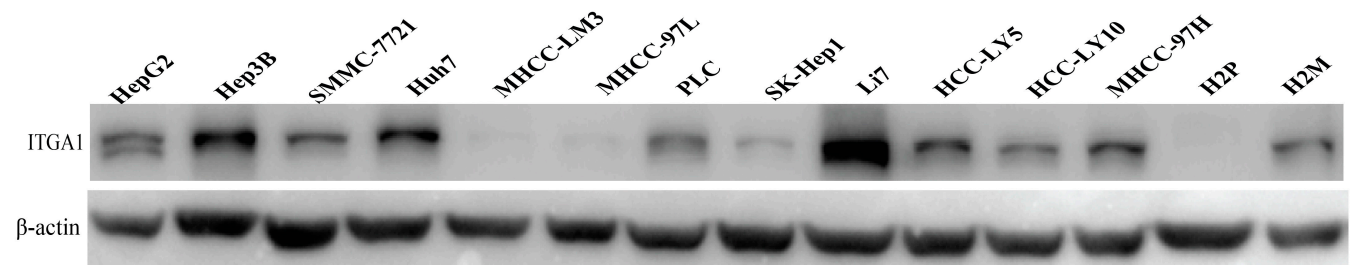

Figure 1. Derivate isocorydine (d-ICD) inhibits hepatocellular carcinoma (HCC) cell migration and invasion and ITGA1 expression. (A) Various HCC cell lines were incubated with d-ICD, and the wound-healing assay was performed to analyze the migration ability (scale bar stand for $200 \mu \mathrm{m}$ ); (B) The Trans-well assay was performed to analyze the invasion ability of HCC cells after d-ICD treatment (scale bar stand for $100 \mu \mathrm{m}$ ); (C) RT-PCR and western blotting analyses were performed to examine ITGA1 expression in HCC cells with d-ICD treatment for $0 \mathrm{~h}, 12 \mathrm{~h}$, and $24 \mathrm{~h}$; (D) Western blot analyzed endogenous ITGA1 expression in HCC cells. $\left({ }^{*} p<0.05,{ }^{* *} p<0.01\right)$.

\subsection{Overexpression of ITGA1 Promotes HCC Cell Migration and Invasion In Vitro}

To further investigated the role of ITGA1 in HCC, we selected the HCC cell lines MHCC-97L, MHCC-LM3, and HCC-LY10, which show intrinsically lower expression of ITGA1, to reconstruct ITGA1 stably overexpression in HCC cells by using ITGA1 lentiviral infection and control lentivirus EX-NEG, also known as E6. The overexpression efficiency was confirmed using qRT-PCR and western blotting analyses (Figure 2A). We examined the function of ITGA1 on HCC cells, and the results of the wound healing assay and trans-well invasion assay demonstrated that overexpression of ITGA1 promoted HCC cell migration and invasion in vitro (Figure 2B,C). 


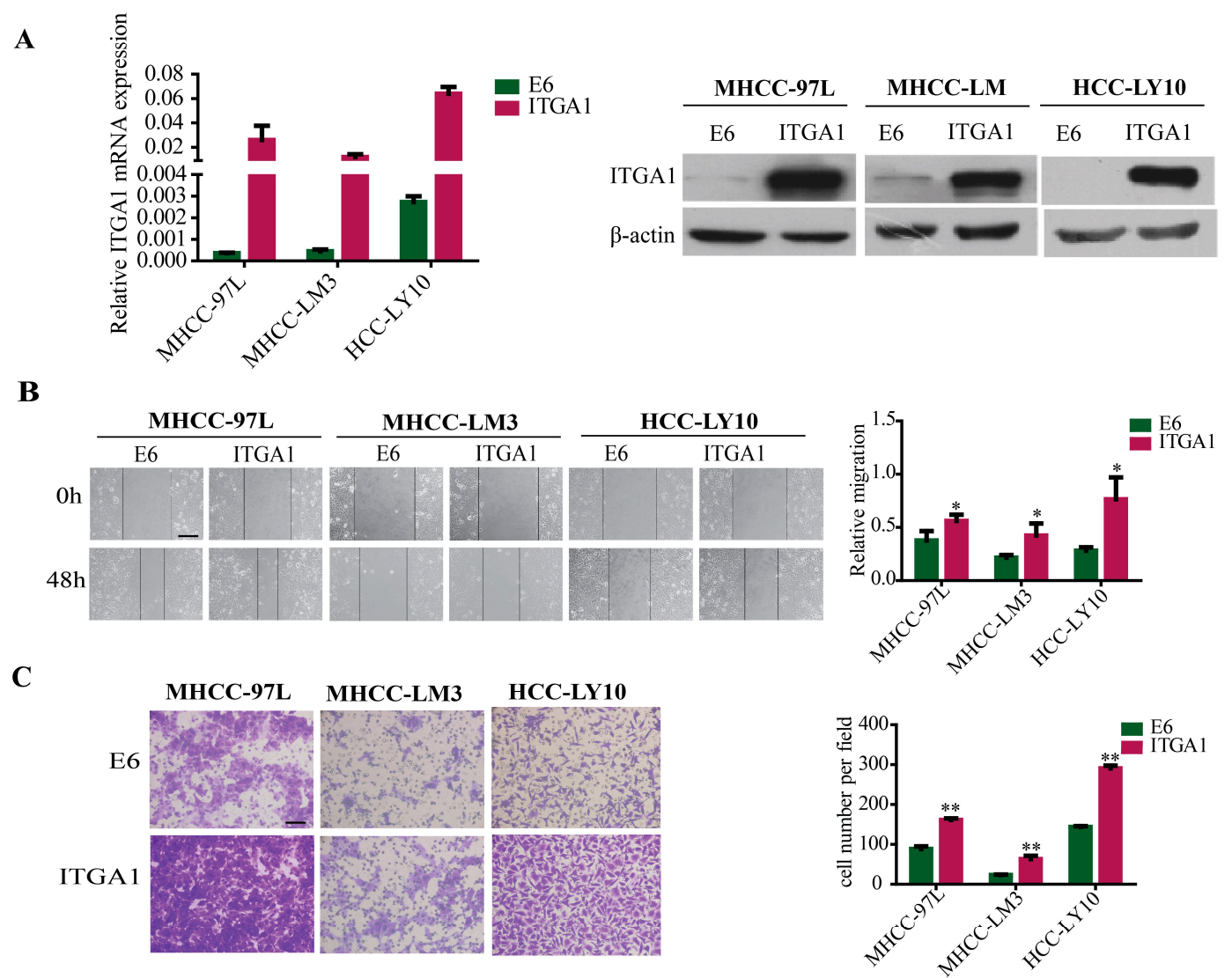

Figure 2. Overexpression of ITGA1 promotes HCC cell migration and invasion in vitro. (A) The efficiency of ITGA1 overexpression were analyzed via RT-PCR and western blot; (B) The in vitro migration ability of MHCC-97L, MHCC-LM3 and HCC-LY10 cells stably transfected with ITGA1 or vector were assessed using the wound-healing assay (scale bar stand for $200 \mu \mathrm{m}$ ); (C) The in vitro invasion ability of those ITGA1-overexpressed HCC cells were assessed using the trans-well assay (scale bar stand for $100 \mu \mathrm{m}) .\left({ }^{*} p<0.05,{ }^{* *} p<0.01\right)$.

\subsection{Silencing ITGA1 Expression Inhibits HCC Cell Migration and Invasion In Vitro}

Conversely, we selected the HCC cell lines SMMC-7721, Huh7, and HCC-LY5, which intrinsically express higher levels of ITGA1 to knock down endogenous ITGA1 expression via shRNA and control lentiviral infection. The ITGA1 silencing efficiency was confirmed by qRT-PCR and western blotting analyses (Figure 3A). Similarly, we performed basic function assays to analyze the ITGA1 function in HCC cells and found that ITGA1 knockdown inhibited HCC cell migration and invasion (Figure 3B,C). Furthermore, to study whether the expression of ITGA1 affecting the expression of ITGB1, we detected ITGB1 expression and found that there was no change in the expression of ITGB1 in ITGA1-overexpressed or ITGA1-silenced expression of HCC cells (Figure S2), which was consistent with a previous report by Pozzi A [25]. 


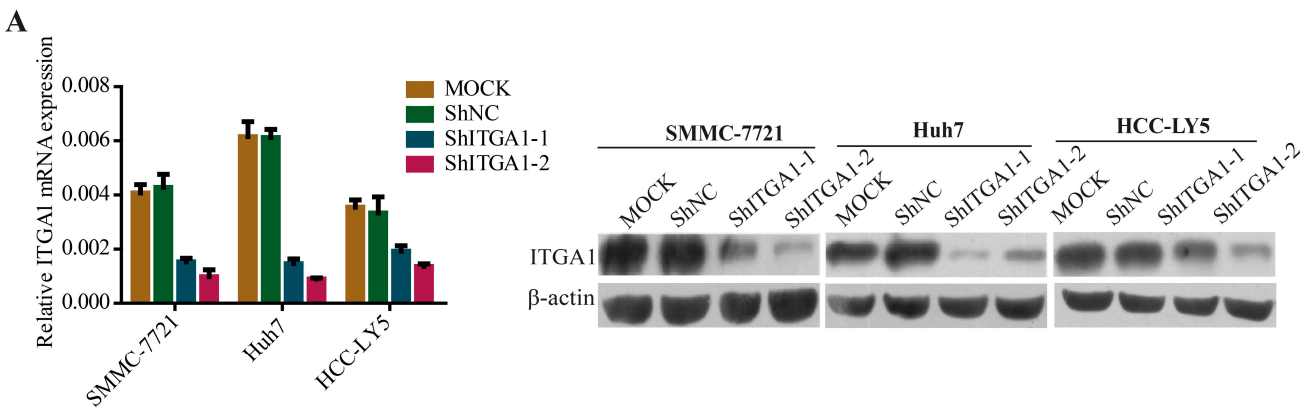

B

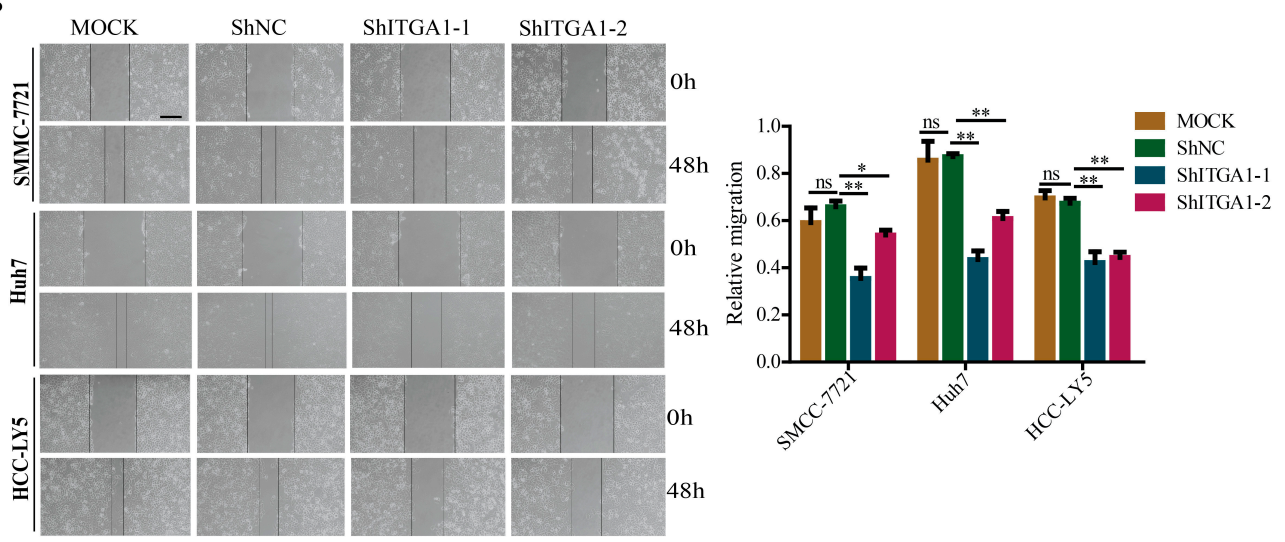

C
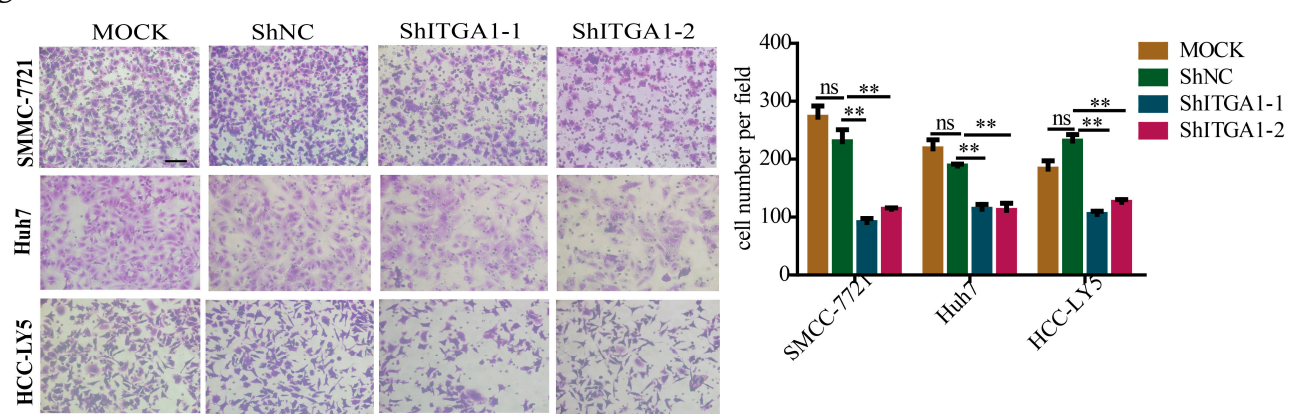

Figure 3. Silencing ITGA1 expression inhibits HCC cell migration and invasion in vitro. (A) qRT-PCR and western blot analyzed the silencing efficiency of ITGA1 expression; (B) The in vitro migration ability of SMMC-7721, Huh7 and HCC-LY5 cells stably transfected with ITGA1 ShRNA or ShNC or MOCK cells were assessed using wound-healing assay (scale bar stand for $200 \mu \mathrm{m}$ ); (C) The in vitro invasion ability of those ITGA1-silencing HCC cells were assessed by trans-well assay (scale bar stand for $100 \mu \mathrm{m}$ ). ("ns" indicates no statistical significance, ${ }^{*} p<0.05,{ }^{* *} p<0.01$ ).

\subsection{Overexpression of ITGA1 Partly Rescues d-ICD-Induced Migration and Invasion Inhibition in HCC Cells}

To determine whether the inhibition of HCC migration and invasion by d-ICD could be dependent on its inhibition on ITGA1 expression, we performed functional compensation assays (Figure 4A,B). Similar results were observed that following d-ICD treatment, the migration and invasion ability of HCC cells was decreased. ITGA1-overexpressed HCC cells moved and invaded faster than E6 control group HCC cells following d-ICD treatment. The migration and invasion ability of HCC cells with ITGA1 overexpression and d-ICD treatment showed no obvious difference when compared with empty HCC cells, suggesting that ITGA1 overexpression partially abrogated the inhibition of d-ICD on migration and invasion of HCC cells. On the basis of previous results, we concluded that d-ICD inhibits HCC cell migration and invasion partly by downregulating ITGA1 expression. 
A

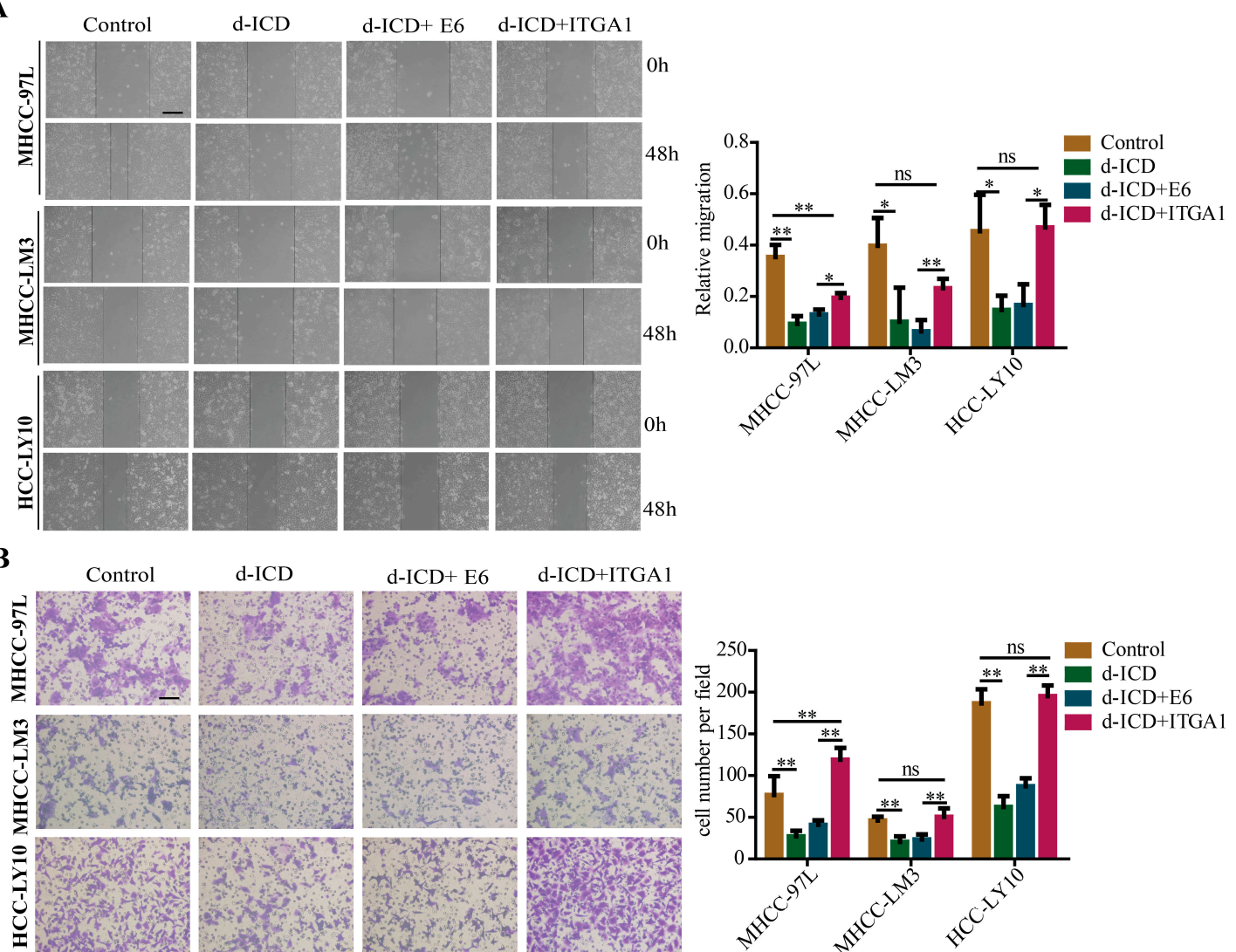

Figure 4. ITGA1 partly rescues d-ICD-induced migration and invasion suppression in HCC cells; (A) The wound-healing assay was performed to detect the migration ability of MHCC-97L, MHCC-LM3 and HCC-LY10 empty cells after treating with or without d-ICD, and MHCC-97L, MHCC-LM3, and HCC-LY10 stably transfected with ITGA1 or vector after treating with d-ICD (scale bar stand for $200 \mu \mathrm{m})$; (B) The Trans-well assay was performed to detect the invasion ability of those HCC cells (scale bar stand for $100 \mu \mathrm{m}$ ). ("ns" indicates no statistical significance, ${ }^{*} p<0.05,{ }^{* *} p<0.01$ ).

\subsection{E2F1 Upregulates ITGA1 Expression and d-ICD Inhibits E2F1 Expression in HCC Cells}

To further study the regulation of ITGA1 in HCC cells, the ITGA1 promoter was analyzed via transcriptional factors binding predicted websites TFBIND, ALGGEN-PROMO, GENE REGULATION, and QIAGEN. All of the four websites predicted that the E2F1 binding site was located at $-330 \mathrm{bp} /-315 \mathrm{bp}$ of the ITGA1 promoter (Figure 5A). We further found that the expression of ITGA1 was upregulated in E2F1 overexpression HCC cells (Figure 5D), and was downregulated in E2F1 silencing HCC cells (Figure S3). Meanwhile, we observed that the expression of ITGA1 positively related to the expression of E2F1 in HCC tissue (Figure S4). Based on the prediction and expression analysis of $E 2 F 1$ and ITGA1, we hypothesized that $E 2 F 1$ may bind to the ITGA1 promoter and regulate ITGA1 expression. To confirm our hypothesis, we constructed clones of the ITGA1 promoter as well as truncated and mutant variations. Dual luciferase reporter gene studies were performed to detect the activity of the ITGA1 promoter in 293T cells (Figure 5B), and in HCC cells (Figure S5). These results revealed that E2F1 increased ITGA1 promoter activity. However, E2F1 did not increase ITGA1 promoter activity containing a putative E2F1-mutated binding site (Figure 5B). Binding of $E 2 F 1$ to the ITGA1 promoter was further confirmed using a chromatin immunoprecipitation assay (Figure 5C). Meanwhile, we found that the expression of E2F1 was downregulated after d-ICD treatment in HCC cells (Figure 5E), which prompted d-ICD suppression of HCC cell migration and invasion may partly via the E2F1/ITGA1 pathway. 
A

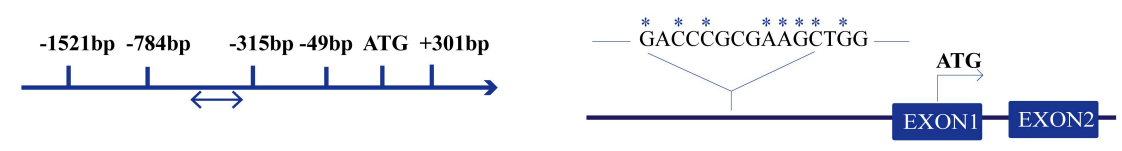

B
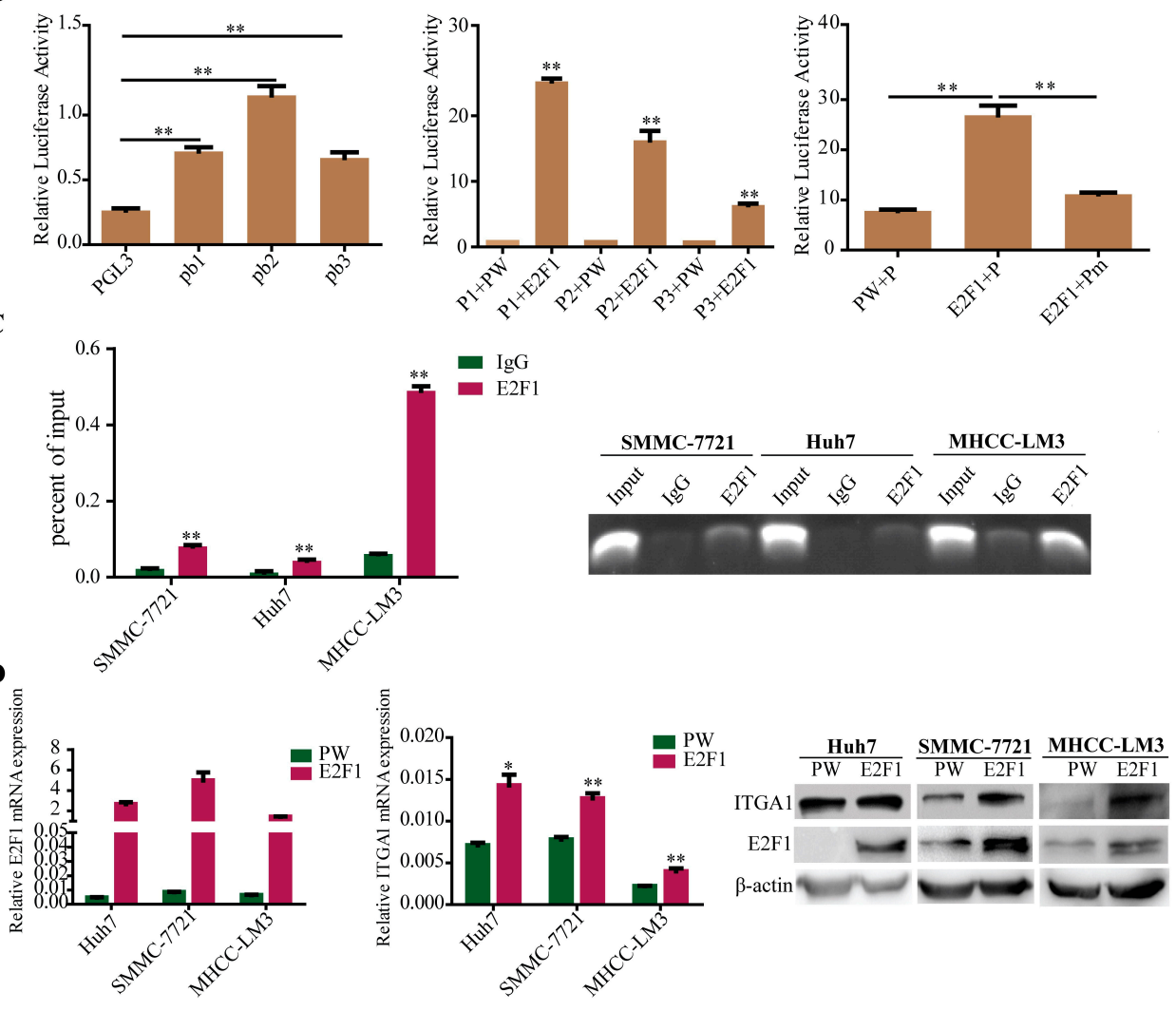

E

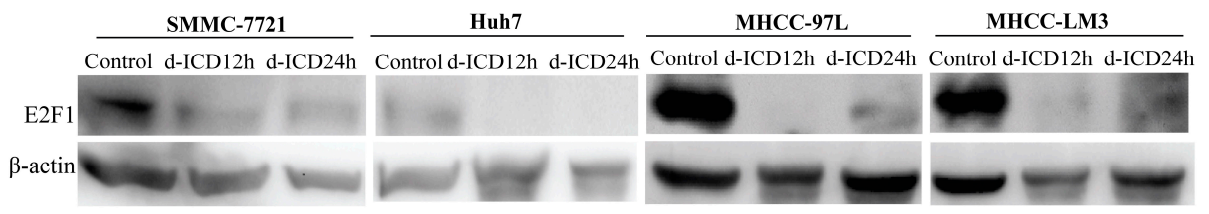

Figure 5. E2F1 transcriptional upregulates ITGA1 expression and d-ICD inhibits E2F1 expression in HCC cells. (A) Identification of potential E2F1 binding sites (double arrow marked) in the ITGA1 promoter (arrow direction indicates sequence from $5^{\prime}$ to $3^{\prime}$ ) according to four TF-binding prediction websites (QIAGEN, ALGGEN PROMO, TFBIND, GENE REGULATION), and the mutant sites are marked (arrow direction indicates transcriptional direction of the downstreem gene); (B) Dual luciferase reporter gene studies were performed to detect the ITGA1 promoter and its truncated and mutant construct activity in 293T cells; (C) Binding of E2F1 to the ITGA1 promoter in HCC cells was analyzed by chromatin immunoprecipitation. qRT-PCR was used to analyze the ITGA1 promoter, and agarose gel electrophoresis was used to analyze the crosslinking status; (D) qRT-PCR and western blotting analyzed E2F1 and ITGA1 expression in HCC cells with transiently transfected E2F1 plasmid; (E) Western blotting analyses of E2F1 expression in HCC cells treated with d-ICD at different time points. $\left({ }^{*} p<0.05, \mathrm{~m}\right)$.

\section{Discussion}

It is well known that hepatocellular carcinoma greatly threatens human health due to the high morbidity and mortality and the cure difficulty. Thus, it is urgent to develop an effective drug for HCC treatment. In our previous studies, d-ICD has proven to be an effective anti-HCC agent due to its effects as an anti-proliferation and anti-drug-resistant agent [6,7]. In the present study, we continued to probe the function and relevant molecular mechanisms of d-ICD in HCC cells. We initially found 
that d-ICD inhibited HCC cell migration and invasion in vitro. Though d-ICD mildly induced HCC cell apoptosis during migration assay (Figure S6), but we still believe there must be another molecular mechanism during the process of d-ICD inhibiting HCC cell migration and invasion. So, we screened potential metastasis-related differential expression genes from the cDNA microarrays of Huh7 and SMMC-7721 cells between the control and d-ICD treatment groups [7], and due to our knowledge of its gene biological function, we selected the target gene ITGA1. Next, we cultured HCC cells with $\mathrm{d}-\mathrm{ICD}$ and confirmed the decreased expression of ITGA1 at the mRNA and protein levels.

Integrin $\alpha 1$ coupled with integrin $\beta 1$ form a heterodimer located at the cell membrane [26]. Pozzi's team has studied the function of integrin $\alpha 1$ in various diseases for many years and found that integrin $\alpha 1$ plays an important role in proliferation, adhesion, migration, and angiogenesis [26]. Many reports showed that ITGA1 may play an important role on migration and invasion in various tumor [15-17,27]. In addition, Wan, et al found that overexpression of ITGA1 in HCC cells presented high metastasis potential in the lymph gland [28]. To verify the migration and invasion inhibition of d-ICD to HCC cell partially by downregulating ITGA1 expression, we further investigated the function of ITGA1 in HCC cells. Consistent with the suggested role of ITGA1 as a potential tumor promoter, we showed that the migration and invasion ability of HCC cells was enhanced when ITGA1 was stably overexpressed, while the migration and invasion ability of HCC cells was decreased when ITGA1 expression was stably knocked down. However, ITGA1 has no obvious effect on HCC cell proliferation or apoptosis in vitro (Figure S7). The results of both overexpression and decreased expression of ITGA1 indicated that ITGA1 plays a critical role on HCC cell migration and invasion. Furthermore, the results of functional compensation experiments showed that ITGA1 overexpression partially rescued the migration and invasion suppression of d-ICD in HCC cells. So far, based on these findings, we concluded that d-ICD inhibits HCC cell migration and invasion partly by down-regulating ITGA1 expression.

Furthermore, we explored the expression regulation of ITGA1 in HCC cells. Based on the prediction of the transcription factor website and our experimental results, we found that $E 2 F 1$ directly binds to the ITGA1 promoter and that the expression of ITGA1 positively correlated to the expression of E2F1 in HCC cells and tissue, suggesting that $E 2 F 1$, the powerful transcription factor, also transcriptionally upregulates ITGA1 expression in HCC. There have been previous reports demonstrating that E2F1 mediates tumor metastatic dissemination by up-regulating fibronectin [29]. In HCC, E2F1 expression and its effects have been showed to be controversial during tumorigenesis [24,30,31]. Moreover, we found that E2F1 is downregulated in HCC cells following d-ICD treatment, indicating that d-ICD inhibited ITGA1 expression may partly through downregulating E2F1 expression. These findings at least partially explains molecular mechanisms underlying action of d-ICD in inhibiting migration and invasion of HCC in vitro.

It is important that combination drugs to exert synergistic effect during routine chemotherapy. Isocorydine (ICD) has been reported to enhance the sensitivity of HCC cell lines to DXR by targeting HCC SP cells in our previous study [3]. Sorafenib, as an only one anti-HCC drug in clinics approved by FDA, was demonstrated to be more effective when combination administration with low dosage of d-ICD [2]. The function of d-ICD inhibiting HCC cells drug resistance may serve as the foundation of d-ICD being an adjuvant during anti-HCC chemotherapy. Therefore, we consider that d-ICD may improve the efficacy of many other chemotherapeutic drugs by enhancing the cytotoxic effect on HCC cells. Continuing studies are needed.

\section{Materials and Methods}

\subsection{Cell Lines and Cell Culture}

The human HCC cell lines SMMC-7721 was used in our study and were supplied by the Cell Bank of the Institute of Biochemistry and Cell Biology, China Academy of Sciences (Shanghai, China) in 2007. The cell lines MHCC-97L and MHCC-LM3 were kindly provided by the Liver Cancer Institute of Zhongshan Hospital, Fudan University (Shanghai, China) in 2007, and human HCC-LY5 and 
HCC-LY10 were established in our laboratory in 2011, which was authenticated by ourselves. 293T was purchased from the American Type Culture Collection (Manassas, VA, USA), and Huh7 cells were obtained from the Riken Cell Bank (Tsukuba, Japan) in 2010. The cell nutrient solution for all of the above cells was Dulbecco's modified Eagle's medium (DMEM) (Sigma-Aldrich, St. Louis, MO, USA) containing $10 \%$ fetal bovine serum (FBS) (HyClone), which was heat-inactivated at $56{ }^{\circ} \mathrm{C}$ for $30 \mathrm{~min} ; 100 \mathrm{IU} / \mathrm{mL}$ penicillin G; and $100 \mu \mathrm{g} / \mathrm{mL}$ streptomycin (Sigma-Aldrich). All cell lines were incubated at $37{ }^{\circ} \mathrm{C}$ in a humidified atmosphere with $5 \% \mathrm{CO}_{2}$. All cell lines used in this study were thawed fresh every two months and used within 20 passages. These cell lines were mycoplasma-free and authenticated by their examination of morphology and growth profile.

\subsection{Western Blotting Analyses}

Protein sample extraction and concentration detection were performed as previously described [6], and protein were separated onto $10 \%$ SDS-PAGE and transferred onto nitrocellulose or polyvinylidene difluoride (PVDF) membranes. Next, the membranes were blocked with $5 \%$ nonfat milk for $1 \mathrm{~h}$ at room temperature and incubated with primary antibodies (integrin $\alpha 1$ BAF 5676 R\&D system, integrin $\beta 1$ SC-59829, E2F1 SC-251) overnight at $4{ }^{\circ} \mathrm{C}$. After washing with $1 \times$ PBST 3 times, $10 \mathrm{~min} /$ each, a HRP-conjugated secondary antibody was added for $1 \mathrm{~h}$ at room temperature, and $\beta$-actin (A3854 Sigma-Aldrich) was used as a loading control. Chemiluminescence was detected using the Super Signal West Femto Chemiluminescent substrate kit (Thermo scientific, Waltham, MA, USA). Informed consent was obtained from all patients, and the study was approved by the Ethic Committee of Shanghai Jiao Tong University. Information on all of the antibodies used in this study is provided in Table S1.

\subsection{Quantitative Real-Time Polymerase Chain Reaction ( $q R T-P C R$ )}

Total RNA of cell lines was extracted using TRIzol reagent (Invitrogen, Carlsbad, CA, USA) and then reverse transcribed into cDNA using the PrimeScript ${ }^{\mathrm{TM}} \mathrm{RT}$ Reagent Kit (TaKaRa Biotechnology, Shimogyo-ku, Japan). Real-time PCR was performed using SYBR Premix Ex Taq (TaKaRa Biotechnology) according to the manufacturer's protocol. The primers used to quantify the target genes or DNA fragments are provided in Table S2.

\subsection{Plasmid Construction, Lentivirus Production, and Cell Transfection}

Full-length human ITGA1 plasmid and empty vector E6 were purchased from GeneCopoeia Company (Guangzhou, China), and the shRNA targeting ITGA1 were designed by Genechem (Shanghai, China) and the sequence is provided in Table S3. The promoter sequences of ITGA1 were cloned into PGL3-Basic (purchased from Promega, Madison, WI, USA) at the KpnI and HindIII sites. The full-length human E2F1 gene coding sequence was PCR-amplified and cloned into pWPXL (Addgene, Cambridge, MA, USA) at the BamHI and EcoRI sites. The primers of the clones and promoter sequence are provided in Tables S4 and S5. Viral packaging and cell transfections were performed as previously described [32].

\subsection{Wound Healing Assay}

Approximately $1 \times 10^{6} \mathrm{HCC}$ cells were seeded onto 6-well plates, and after the cells were cultured overnight and overspread on the plates, we scratched a vertical long wound onto the cells and obtained images at $0 \mathrm{~h}$ and $48 \mathrm{~h}$. Before we obtained the images, we washed the cells twice with $1 \times \mathrm{PBS}$ and replaced the medium with medium containing $2 \%$ FBS or other medium containing d-ICD. The cells were synchronized with $1 \mathrm{mM}$ thymidine (Sigma-Aldrich). Cells that migrated into the wound area were calculated as follows: ( $(0 \mathrm{~h}$ wound wide subtract $48 \mathrm{~h}$ wound wide) $/ 0 \mathrm{~h}$ wound wide). 


\subsection{Trans-Well Invasion Assay}

The trans-well insert (8-mm pore; Merck Millipore, Billerica, MA, USA) was pre-coated with $20 \%$ matrigel (BD Bioscience, San Jose, CA, USA) for $30 \mathrm{~min}$ at $37^{\circ} \mathrm{C}$. A total of $2 \times 10^{5}$ cells were seeded onto the upper chamber of the trans-well in serum-free media, while the bottom chamber of the trans-well was filled with complete medium with or without d-ICD. After $24 \mathrm{~h}$ of incubation, the cells were fixed with $10 \%$ formaldehyde for $30 \mathrm{~min}$ and stained with crystal violet solution. Invasive cells were quantified using ImageJ software.

\subsection{Dual Luciferase Reporter Assay}

First, 293T cells were seeded onto 96-well culture plates for $16 \mathrm{~h}$ and then transiently co-transfected with ITGA1 promoters and PRL-TK and the relevant plasmid. After $48 \mathrm{~h}$ of incubation, Renilla and firefly luciferase activity was determined according to the manufacturer's instructions (Promega).

\subsection{Chromatin Immunoprecipitation Assay (ChIP)}

Chromatin immunoprecipitation assays (ChIP) were performed as previously described [33].

\subsection{Cell Apoptosis Analysis}

Cells were digested, and washed twice with $1 \times$ PBS, then resuspended in binding buffer to a concentration of $1 \times 10^{6}$ cells $/ \mathrm{mL}$. Cell suspensions $(500 \mu \mathrm{L})$ were added to tubes, then added $5 \mathrm{uL}$ of Annexin V FITC conjugate and $10 \mu \mathrm{L}$ of Propidium Iodide Solution (both available from Sigma-Aldrich) and incubated at room temperature for $10 \mathrm{~min}$ and protected from light. Fluorescence of the cells were analyzed by flow cytometry.

\subsection{Cell Proliferation Assays}

For MTT assays, 1000-3000 cells per well were plated on 96-well plates and incubated for $16 \mathrm{~h}$. Then, $100 \mu \mathrm{L}$ of MTT reagent (5 mg/mL, Sigma-Aldrich) was added to each well and incubated for $4 \mathrm{~h}$ at $37^{\circ} \mathrm{C}$. The optical density (OD) value was recorded at a dual wavelength $(570 \mathrm{~nm}$ ) every day for 7 days. For colony formation assays, 1000-3000 cells per well were plated on 6-well plates and cultured for 2 weeks, then fixed with $10 \%$ formaldehyde for $30 \mathrm{~min}$ at $37^{\circ} \mathrm{C}$. The cells were stained with Giemsa solution. Finally, the cell colonies were quantified.

\subsection{Statistical Analysis}

All data were presented as the mean \pm standard deviation (SD). Differences between groups were assessed using Student's $t$-test. Differences were considered statistically significant at $p<0.05$. Analyses were performed using Prism 5.0 (GraphPad, San Diego, CA, USA).

\section{Conclusions}

In summary, the present study is the first study to demonstrate that d-ICD inhibits the migration and invasion ability of HCC cells in vitro. Furthermore, ITGA1 is an important target of d-ICD and is involved in the effects of d-ICD on migration and invasion in HCC cells. Moreover, d-ICD downregulates ITGA1 expression may partly through suppressing the expression of E2F1 in HCC cells. Taken together, our observations help to better understand the effect and mechanism of d-ICD treatment in HCC cells as well as the function of ITGA1 in HCC. More valuable findings regarding d-ICD in HCC treatment should be uncovered in future studies.

Supplementary Materials: Supplementary materials can be found at www.mdpi.com/1422-0067/18/3/514/s1.

Acknowledgments: This work was supported in part by grants from the National Natural Science Foundation of China (81472726), the National Key Program for Basic Research of China (973) (2015CB553905), the National Key Sci-Tech Special Project of China (2013ZX10002-011), the Key Discipline and Specialty Foundation of Shanghai Municipal Commission of Health and Family Planning, and the SKLORG Research foundation (91-15-03). 
Author Contributions: Jinjun Li conceived of and designed the experiments; Xiaoqin Liu, Hua Tian, Chao Ge, Hong Li, Fangyu Zhao, and Ming Yao performed the experiments; Xiaoqin Liu analyzed the data and wrote the paper.

Conflicts of Interest: The authors declare no conflict of interest.

\section{References}

1. Torre, L.A.; Bray, F.; Siegel, R.L.; Ferlay, J.; Lortet-Tieulent, J.; Jemal, A. Global cancer statistics, 2012. Cancer J. Clin. 2015, 65, 87-108. [CrossRef] [PubMed]

2. Marquardt, J.U.; Thorgeirsson, S.S. SnapShot: Hepatocellular carcinoma. Cancer Cell 2014, 25, 550. [CrossRef] [PubMed]

3. Sun, H.; Hou, H.; Lu, P.; Zhang, L.; Zhao, F.; Ge, C.; Wang, T.; Yao, M.; Li, J. Isocorydine inhibits cell proliferation in hepatocellular carcinoma cell lines by inducing G2/M cell cycle arrest and apoptosis. PLOS ONE 2012, 7, e36808. [CrossRef] [PubMed]

4. Lu, P.; Sun, H.; Zhang, L.; Hou, H.; Zhang, L.; Zhao, F.; Ge, C.; Yao, M.; Wang, T.; Li, J. Isocorydine targets the drug-resistant cellular side population through PDCD4-related apoptosis in hepatocellular carcinoma. Mol. Med. 2012, 18, 1136-1146. [CrossRef] [PubMed]

5. Zhong, M.; Liu, Y.; Liu, J.; Di, D.; Xu, M.; Yang, Y.; Li, W.; Chen, Y.; Liu, J. Isocorydine derivatives and their anticancer activities. Molecules 2014, 19, 12099-12115. [CrossRef] [PubMed]

6. Chen, L.; Tian, H.; Li, M.; Ge, C.; Zhao, F.; Zhang, L.; Li, H.; Liu, J.; Wang, T.; Yao, M.; et al. Derivate isocorydine inhibits cell proliferation in hepatocellular carcinoma cell lines by inducing G2/M cell cycle arrest and apoptosis. Tumour Biol. 2016, 37, 5951-5961. [CrossRef] [PubMed]

7. Li, M.; Zhang, L.; Ge, C.; Chen, L.; Fang, T.; Li, H.; Tian, H.; Liu, J.; Chen, T.; Jiang, G.; et al. An isocorydine derivative (d-ICD) inhibits drug resistance by downregulating IGF2BP3 expression in hepatocellular carcinoma. Oncotarget 2015, 6, 25149-25160. [CrossRef] [PubMed]

8. Barczyk, M.; Carracedo, S.; Gullberg, D. Integrins. Cell Tissue Res. 2010, 339, 269-280. [CrossRef] [PubMed]

9. Virtanen, I.; Korhonen, M.; Kariniemi, A.L.; Gould, V.E.; Laitinen, L.; Ylanne, J. Integrins in human cells and tumors. Cell Differ. Dev. 1990, 32, 215-227. [CrossRef]

10. Schadendorf, D.; Gawlik, C.; Haney, U.; Ostmeier, H.; Suter, L.; Czarnetzki, B.M. Tumour progression and metastatic behaviour in vivo correlates with integrin expression on melanocytic tumours. J. Pathol. 1993, 170, 429-434. [CrossRef] [PubMed]

11. Sanders, R.J.; Mainiero, F.; Giancotti, F.G. The role of integrins in tumorigenesis and metastasis. Cancer Investig. 1998, 16, 329-344. [CrossRef] [PubMed]

12. Zallocchi, M.; Johnson, B.M.; Meehan, D.T.; Delimont, D.; Cosgrove, D. $\alpha 1 \beta 1$ integrin/Rac1-dependent mesangial invasion of glomerular capillaries in Alport syndrome. Am. J. Pathol. 2013, 183, 1269-1280. [CrossRef] [PubMed]

13. Senger, D.R.; Perruzzi, C.A.; Streit, M.; Koteliansky, V.E.; de Fougerolles, A.R.; Detmar, M. The $\alpha 1 \beta 1$ and $\alpha 2 \beta 1$ integrins provide critical support for vascular endothelial growth factor signaling, endothelial cell migration, and tumor angiogenesis. Am. J. Pathol. 2002, 160, 195-204. [CrossRef]

14. Zhang, R.; Wang, N.; Zhang, M.; Zhang, L.N.; Guo, Z.X.; Luo, X.G.; Zhou, H.; He, H.P.; Zhang, T.C. Rho/MRTF-A-induced integrin expression regulates angiogenesis in differentiated multipotent mesenchymal stem cells. Stem Cells Int. 2015, 2015, 534758. [CrossRef] [PubMed]

15. Schadendorf, D.; Fichtner, I.; Makki, A.; Alijagic, S.; Kupper, M.; Mrowietz, U.; Henz, B.M. Metastatic potential of human melanoma cells in nude mice-Characterisation of phenotype, cytokine secretion and tumour-associated antigens. Br. J. Cancer 1996, 74, 194-199. [CrossRef] [PubMed]

16. Gulubova, M.V. Collagen type IV, laminin, $\alpha$-smooth muscle actin ( $\alpha \mathrm{SMA}), \alpha 1$ and $\alpha 6$ integrins expression in the liver with metastases from malignant gastrointestinal tumours. Clin. Exp. Metastasis 2004, 21, $485-494$. [CrossRef] [PubMed]

17. Boudjadi, S.; Carrier, J.C.; Beaulieu, J.F. Integrin $\alpha 1$ subunit is up-regulated in colorectal cancer. Biomark. Res. 2013, 1, 16. [CrossRef] [PubMed]

18. Fukuda, K.; Saikawa, Y.; Yagi, H.; Wada, N.; Takahashi, T.; Kitagawa, Y. Role of integrin $\alpha 1$ subunits in gastric cancer patients with peritoneal dissemination. Mol. Med. Rep. 2012, 5, 336-340. [PubMed] 
19. Chen, X.; Su, Y.; Fingleton, B.; Acuff, H.; Matrisian, L.M.; Zent, R.; Pozzi, A. An orthotopic model of lung cancer to analyze primary and metastatic NSCLC growth in integrin $\alpha 1$-null mice. Clin. Exp. Metastasis 2005, 22, 185-193. [CrossRef] [PubMed]

20. Macias-Perez, I.; Borza, C.; Chen, X.; Yan, X.; Ibanez, R.; Mernaugh, G.; Matrisian, L.M.; Zent, R.; Pozzi, A. Loss of integrin $\alpha 1 \beta 1$ ameliorates Kras-induced lung cancer. Cancer Res. 2008, 68, 6127-6135. [CrossRef] [PubMed]

21. Cam, H.; Dynlacht, B.D. Emerging roles for E2F: Beyond the G1/S transition and DNA replication. Cancer Cell 2003, 3, 311-316. [CrossRef]

22. La Thangue, N.B. The yin and yang of E2F-1: Balancing life and death. Nat. Cell Biol. 2003, 5, 587-589. [CrossRef] [PubMed]

23. Jiang, H.; Martin, V.; Gomez-Manzano, C.; Johnson, D.G.; Alonso, M.; White, E.; Xu, J.; McDonnell, T.J.; Shinojima, N.; Fueyo, J. The RB-E2F1 pathway regulates autophagy. Cancer Res. 2010, 70, 7882-7893. [CrossRef] [PubMed]

24. Palaiologou, M.; Koskinas, J.; Karanikolas, M.; Fatourou, E.; Tiniakos, D.G. E2F-1 is overexpressed and pro-apoptotic in human hepatocellular carcinoma. Int. J. Pathol. 2012, 460, 439-446. [CrossRef] [PubMed]

25. Pozzi, A.; Moberg, P.E.; Miles, L.A.; Wagner, S.; Soloway, P.; Gardner, H.A. Elevated matrix metalloprotease and angiostatin levels in integrin $\alpha 1$ knockout mice cause reduced tumor vascularization. Proc. Natl. Acad. Sci. USA 2000, 97, 2202-2207. [CrossRef] [PubMed]

26. Gardner, H. Integrin $\alpha 1 \beta 1$. Adv. Exp. Med. Biol. 2014, 819, 21-39. [PubMed]

27. Yasoshima, T.; Denno, R.; Kawaguchi, S.; Sato, N.; Okada, Y.; Ura, H.; Kikuchi, K.; Hirata, K. Establishment and characterization of human gastric carcinoma lines with high metastatic potential in the liver: Changes in integrin expression associated with the ability to metastasize in the liver of nude mice. Jpn. J. Cancer Res. 1996, 87, 153-160. [CrossRef] [PubMed]

28. Wan, J.; Wen, D.; Dong, L.; Tang, J.; Liu, D.; Liu, Y.; Tao, Z.; Gao, D.; Sun, H.; Cao, Y.; et al. Establishment of monoclonal HCC cell lines with organ site-specific tropisms. BMC Cancer 2015, 15, 678. [CrossRef] [PubMed]

29. Meier, C.; Spitschak, A.; Abshagen, K.; Gupta, S.; Mor, J.M.; Wolkenhauer, O.; Haier, J.; Vollmar, B.; Alla, V.; Putzer, B.M. Association of RHAMM with E2F1 promotes tumour cell extravasation by transcriptional up-regulation of fibronectin. J. Pathol. 2014, 234, 351-364. [CrossRef] [PubMed]

30. Conner, E.A.; Lemmer, E.R.; Omori, M.; Wirth, P.J.; Factor, V.M.; Thorgeirsson, S.S. Dual functions of E2F1 in a transgenic mouse model of liver carcinogenesis. Oncogene 2000, 19, 5054-5062. [CrossRef] [PubMed]

31. Wang, S.N.; Wang, L.T.; Sun, D.P.; Chai, C.Y.; Hsi, E.; Kuo, H.T.; Yokoyama, K.K.; Hsu, S.H. Intestine-specific homeobox (ISX) upregulates E2F1 expression and related oncogenic activities in HCC. Oncotarget 2016, 7, 36924-36939. [CrossRef] [PubMed]

32. Tian, H.; Ge, C.; Li, H.; Zhao, F.; Hou, H.; Chen, T.; Jiang, G.; Xie, H.; Cui, Y.; Yao, M.; et al. Ribonucleotide reductase $\mathrm{M} 2 \mathrm{~B}$ inhibits cell migration and spreading by early growth response protein 1-mediated phosphatase and tensin homolog/Akt1 pathway in hepatocellular carcinoma. Hepatology 2014, 59, 1459-1470. [CrossRef] [PubMed]

33. Fang, T.; Cui, M.; Sun, J.; Ge, C.; Zhao, F.; Zhang, L.; Tian, H.; Zhang, L.; Chen, T.; Jiang, G.; et al. Orosomucoid 2 inhibits tumor metastasis and is upregulated by CCAAT/enhancer binding protein $\beta$ in hepatocellular carcinomas. Oncotarget 2015, 6, 16106-16119. [CrossRef] [PubMed]

(C) 2017 by the authors. Licensee MDPI, Basel, Switzerland. This article is an open access article distributed under the terms and conditions of the Creative Commons Attribution (CC BY) license (http:/ / creativecommons.org/licenses/by/4.0/). 